\title{
Spektroskopische Untersuchung protonenleitfähiger metallorganischer Gerüstverbindungen
}

\author{
D. Klawinski ${ }^{1}$, T. Homburg ${ }^{2}$, N.Stock ${ }^{2}$, M. Tiemann ${ }^{1}$, T. Wagner ${ }^{1}$ \\ 1 Universität Paderborn, Fakultät für Naturwissenschaften, Department Chemie, \\ Warburger Str. 100, D-33098 Paderborn \\ ${ }^{2}$ Christian-Albrechts-Universität zu Kiel, Mathematisch-Naturwissenschaftliche Fakultät, Sektion \\ Chemie, Max-Eyth-Str. 2, D-24118 Kiel \\ Danielle.Klawinski@upb.de
}

\begin{abstract}
Zusammenfassung
Wir stellen erste Ergebnisse der Entwicklung eines Sensorelements zur in situ Überwachung der Feuchte und Protonenleitfähigkeit in neuartigen Protonenaustauschmembranen (PEM, proton exchange membrane) für Brennstoffzellen (FCs) vor. Unter Ausnutzung von Streufeldeffekten (fringing electric fields) sollen später die PEMs im Betrieb über Wechselstrommessungen charakterisiert werden. Die hier vorgestellten Experimente zielen auf die Charakterisierung von neuartigen protonenleitfähigen Materialien, den sogenannten metallorganischen Gerüstverbindungen, mit Hilfe von Impedanzspektroskopie und der Entwicklung geeigneter Ersatzschaltbilder und Modelle zur Auswertung der Messergebnisse. Die metallorganischen Gerüstverbindungen liegen als polykristallines Material in Pulverform vor. Hauptaugenmerk der aktuellen Arbeit ist deshalb die Entwicklung eines geeigneten Aufbaus zur Charakterisierung von kleinen Kristalliten $(<1 \mathrm{~mm})$ insbesondere hinsichtlich von Anisotropieeffekten. Die Impedanzeigenschaften der Materialien werden, entsprechend der später geplanten Anwendung, unter dem Einfluss verschiedener relativen Luftfeuchtigkeiten und Temperaturen erfasst.
\end{abstract}

Keywords: Impedanzspektroskopie,metallorganische Gerüstverbindungen,Kristallite,PEMFC,Protonenleitfähigkeit

\section{Protonenaustauschmembran- Brennstoffzellen}

Die Entwicklung von umweltfreundlichen, günstigen und robusten Verfahren zur Erzeugung und Gewinnung elektrischer Energie hat in den letzten Jahren stark zugenommen. Als Alternative zu konventionellen Energieerzeugern rücken Brennstoffzellen (FCs) immer mehr in den Fokus [1].

Protonenaustauschmembran-Brennstoffzellen (PEMFCs) zeichnen sich durch ihre geringe Betriebstemperatur und eine relativ hohe Energiedichte des Brennstoffs aus. In PEMFCs werden Wasserstoff und Sauerstoff $\mathrm{Zu}$ elektrischer Energie, Wärme und Wasser umgewandelt, wobei beide Gase durch eine protonen-leitfähige Membran getrennt werden. Die Eigenschaften der PEMFCs werden typischerweise durch die Effizienz der verwendeten Protonenaustauschmembran bestimmt. Gängige Membranmaterialien zeigen jedoch starke Abhängigkeiten der Protonenleitfähigkeit von den Betriebsbedingungen, insbesondere der Feuchte. Aus diesem Grund erfolgt eine - meist auf empirischen Ergebnissen basierende Steuerung der verschiedenen Betriebsparameter, wie z.B. die Zuführung von Prozessmedien in Abhängigkeit des Betriebszustands der Brennstoffzelle.

\section{Optimierungsansätze}

Die Möglichkeit zur in situ Überwachung des Membranmaterials eröffnet die Möglichkeit zur Optimierung des Betriebs von PEMFCs.

Dieser Beitrag befasst sich mit zwei Ansätzen zur Verbesserung.

Zum einen soll die Impedanzspektroskopie zur (später tomographischen) Analyse des Feuchtegehalts und der Protonenleitfähigkeit von PEM-Materialien eingesetzt werden. Hierbei ist es möglich, durch die Verwendung 
verschiedener Elektrodengeometrien (fringing electric field), ortsaufgelöste Informationen zu gewinnen.

Zum anderen sollen neuartige Materialien auf Basis von Koordinationspolymeren (CPs; darunter poröse sogenannte metallorganische Gerüstverbindungen, MOFs) als feuchteunabhängige Protonenleiter verwendet werden.

\section{Metallorganische Gerüstverbindungen und Koordinationspolymere}

Als vielversprechende ionen- bzw. protonenleitfähige Materialen eignen sich beispielsweise metallorganische Gerüstverbindungen (MOFs; poröse Koordinationspolymere) $[2,3]$.

MOFs sind dreidimensionale poröse und kristalline Netzwerke, deren anorganische Metallzentren über organische Linkermoleküle verknüpft sind. Es konnte gezeigt werden, dass die Manipulation der lonenleitfähigkeit durch Verwendung geeigneter Linker, die Phosphonat- und/oder Sulfonatgruppen aufweisen, möglich ist [4].

Die in dieser Studie verwendeten LanthanPhosphonatosulfonate wurden über eine Solvothermalsynthese dargestellt und die Kristallstruktur mittels Einkristallröntgenstrukturanalyse bestimmt.

Im Hinblick auf die Verwendung dieser Materialien in Protonenaustauschmembranen ist die Untersuchung von Anisotropieeffekten der Protonenleitfähigkeit von besonderem Interesse [3,5]. Dazu müssen einzelne MOFKristallite hinsichtlich folgender Aspekte untersucht werden: Zum einen muss geklärt werden, ob die Protonenleitfähigkeit abhängig von der Kristallitorientierung ist, und, daraus folgend, ob der Leitungsmechanismus der Protonen über das Porensystem der MOFs oder interpartikulär stattfindet.

\section{Messaufbau zur Impedanzanalyse}

Um Anisotropieeffekte sowie den Einfluss von Temperatur und Feuchte auf einzelne neuartige MOF-Kristallite mittels Impedanzspektroskopie zu untersuchen, wurde folgender Messaufbau verwendet (siehe Abb. 1).

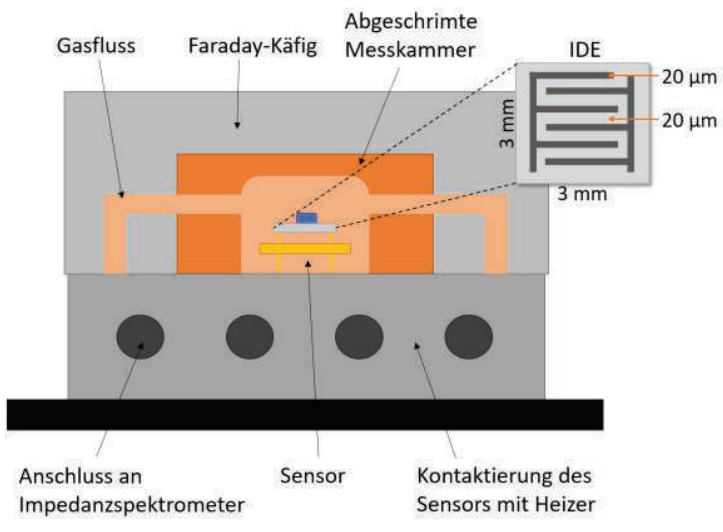

Abb. 1: Schema des Messaufbaus zur Impedanzanalyse einzelner MOFKristallite.

Mit Hilfe des gezeigten Messaufbaus können kommerziell erhältliche Sensorsubstrate mit Interdigitalelektrodenstruktur (IDE) kontaktiert werden. Durch einen Faraday-Käfig, in dem sich der Sensor befindet, ermöglicht der gezeigte Aufbau eine Abschirmung des Sensors zur Verbesserung des Signal-RauschVerhältnisses. Zudem kann die Temperatur auf dem Sensorsubstrat gemessen und ein Gas über den Ein- und Auslass zugeführt werden. Die gewünschte Gaszusammensetzung wird mit Hilfe von Massenflussreglern gesteuert.

Für die Impedanzanalysen spielt die Kontaktierung der MOF-Kristallite eine entscheidende Rolle. Mögliche Ansätze für die Kontaktierung wären: Der Kristallit wird an den Enden entlang der Poren kontaktiert oder nur auf die IDE aufgelegt. Letzterer Ansatz ist in dieser Arbeit gewählt worden, da zur Kontaktierung an den Kristallit-Enden Additive, wie Metalle oder leitfähige Pasten, benötigt werden. Dadurch wird das Porensystem zum einen verschlossen und damit unzugänglich für Gase, zum anderen haben die Zusätze selbst auch Beiträge zur Impedanz.

Das folgende Schema (siehe Abb. 2) veranschaulicht die Position des Kristallits auf der IDE, welches insbesondere für die Untersuchung von Anisotropieeffekten von Bedeutung ist. 


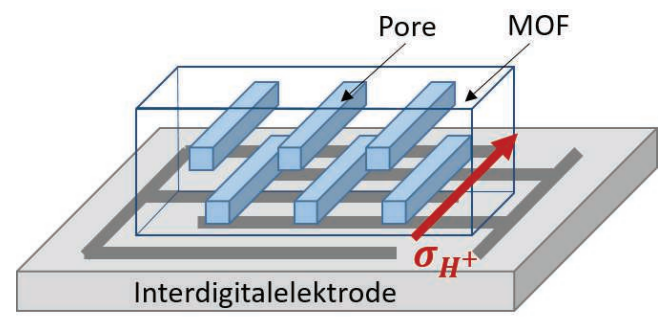

Abb. 2: Schema eines MOF-Kristalliten auf der IDE des Sensorsubstrats. In rot ist die Protonenleitfähigkeit entlang des Porensystems markiert.

Durch diesen Messaufbau können somit die Eigenschaften des Materials ohne Störeinflüsse durch interpartikuläre Kontakte sowie Binder bestimmt werden. Dies ermöglicht zukünftig die systematische Untersuchung verschiedener MOFs, z.B. mit unterschiedlichen Linkermolekülen.

Die Impedanzanalysen wurden mit einem Solartron SI1260 durchgeführt. Zusätzlich wurde ein Transimpedanzverstärker (Chelsea Dielectric Interface) zur Messung der bei den MOF-Kristalliten auftretenden, sehr kleinen Ströme $(p A)$ verwendet.

\section{Ergebnisse}

Im Folgenden werden erste Ergebnisse der Impedanzanalysen einzelner, neuartiger Lanthan-Phosphonatosulfonat-Kristallite gezeigt. Dabei werden zunächst die Einflüsse von variierender relativer Feuchte und Anisotropieeffekte betrachtet.

Zur Untersuchung der Feuchteabhängigkeit der MOF-Kristallite wurden diese mit verschiedenen relativen Feuchten (35 $80 \%$ r.h.) im Stickstoffstrom bei $22{ }^{\circ} \mathrm{C}$ beaufschlagt. Impedanzspektren wurden in einem Frequenzbereich von $1 \mathrm{MHz}-1 \mathrm{~Hz}$ mit einer Amplitude von $0,1 \mathrm{~V}$ nach einer Equilibrierungszeit von $20 \mathrm{~h}$ aufgenommen.

Ein exemplarisches Impedanzspektrum (Nyquist-Plot) der MOF-Kristallite ist in Abb. 3 dargestellt.

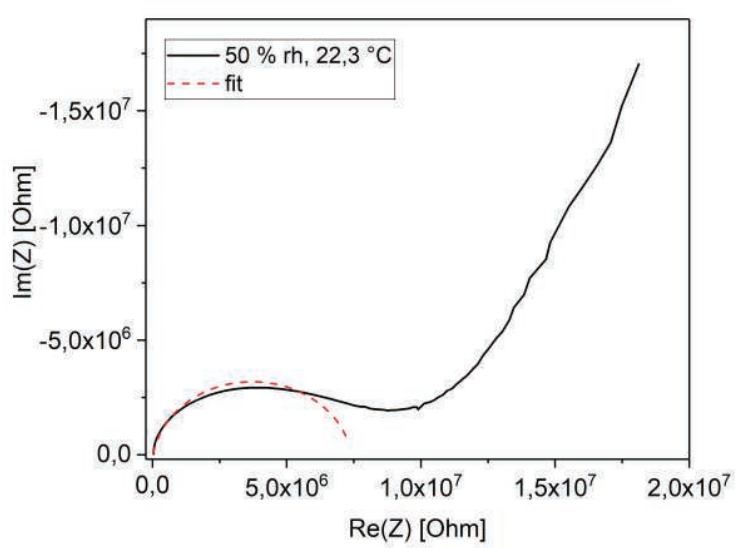

Abb. 3: Exemplarisches Impedanzspektrum einzelner Lanthan-Phosphonatosulfonat-Kristallite (Größe ca. 60$80 \mu \mathrm{m})$ bei $67 \%$ r.h. und $22{ }^{\circ} \mathrm{C}$.

Mit Hilfe eines einfachen Ersatzschaltbildes wurde der Protonenwiderstand in Abhängigkeit von der Umgebungsfeuchte (35-80 \% r.h.) im Stickstoffstrom bei $22{ }^{\circ} \mathrm{C}$ ermittelt (siehe Abb. 5). Zunächst ist in Abb. 4 das Ersatzschaltbild gezeigt.

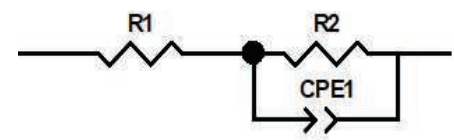

Abb. 4: Ersatzschaltbild zur Ermittlung des Protonenwiderstandes.

Das Ersatzschaltbild gibt den hochfrequenten Bereich des Impedanzspektrums (Halbkreis) wieder. Der Halbkreis wird mit einer Parallelschaltung aus einem Widerstand $R_{2}$ und einem CPE 1 (constant phase element) dargestellt. Vor das R-CPE-Glied wurde ein weiterer Widerstand $R_{1}$ in Serie geschaltet, welcher den Widerstand von Kabeln und Elektroden repräsentiert. Der Widerstand $R_{2}$ wird in der Literatur als Protonenwiderstand angenommen [6]. 


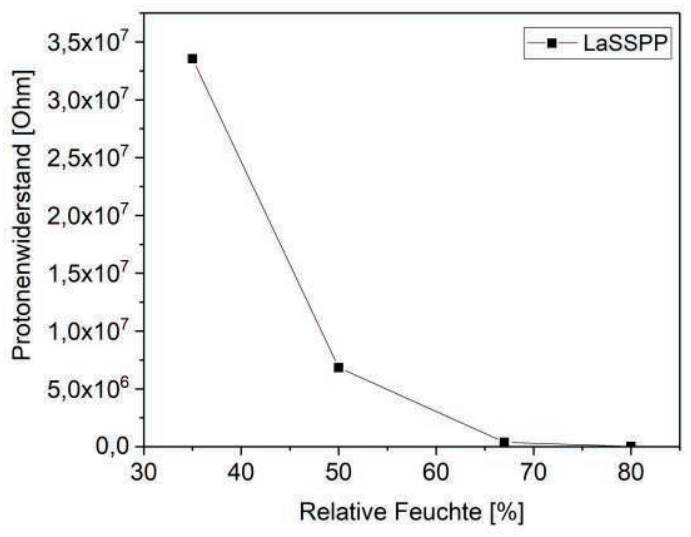

Abb. 5: Protonenwiderstand der LanthanPhosphonatosulfonat-Kristallite in Abhängigkeit der relativen Feuchte.

Wie sich zeigt, weist dieses Material noch nicht die gewünschte Feuchteunabhängigkeit auf (siehe Abb. 5). Der Protonenwiderstand der Kristallite zeigt insbesondere im unteren Bereich (<50\%) eine deutliche Abhängigkeit von der eingestellten relativen Feuchte.

Zur Untersuchung von Anisotropieeffekten der Protonenleitfähigkeit wurden Impedanzspektren in einem Frequenzbereich von $1 \mathrm{MHz}-1 \mathrm{~Hz}$ mit einer Amplitude von $0,1 \mathrm{~V}$ nach einer Equilibrierungszeit von $20 \mathrm{~h}$ bei $80 \%$ relativer Feuchte und $22{ }^{\circ} \mathrm{C}$ aufgenommen.

Die Impedanzspektren (Nyquist-Plot: Bereich der hohen Frequenzen zur besseren Übersicht gezeigt) sind in Abb. 6 dargestellt. Der Kristallit wurde in verschiedenen Positionen (hochkant $H$, waagerecht $W 1$ und waagerecht um $90^{\circ}$ gedreht W2) auf der IDE positioniert. Die fits wurden mit dem oben beschriebenen Ersatzschaltbild (siehe Abb. 4) durchgeführt.

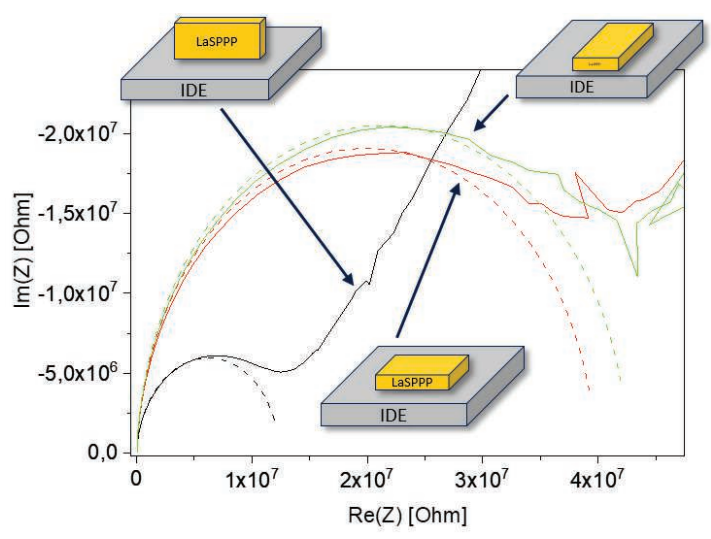

Abb. 6: Impedanzspektren

(hohe Frequenzen) eines LanthanPhosphonatosulfonat-Kristalliten (Größe ca. $300 \mu \mathrm{m}$ ) in verschiedenen Positionen bei $80 \%$ r.h. und $23{ }^{\circ} \mathrm{C}$.

Wie in Abb. 6 erkennbar ist, unterscheiden sich die Impedanzen des Kristallits in den verschiedenen Positionen. Dieses ist besonders deutlich für die Positionen $H$ (hochkant) und W1 (waagerecht).

Mit Hilfe des Ersatzschaltbildes lassen sich die in Tab. 1 zusammengefassten Protonenleitfähigkeiten $\sigma_{H}{ }^{+}$für diesen MOF-Kristalliten in Abhängigkeit von der Position auf der IDE abschätzen.

Tab. 1: Abgeschätzte Protonenleitfähigkeiten in Abhängigkeit von der MOF-KristallOrientierung auf der IDE.

\begin{tabular}{|c|c|c|}
\hline Position & $R_{2}[\mathrm{Ohm}]$ & $\sigma_{H}{ }^{+}[\mathrm{S} / \mathrm{cm}]$ \\
\hline$H$ & $1,2326 \cdot 10^{-7}$ & $2,8 \cdot 10^{-5}$ \\
\hline$W 1$ & $3,9735 \cdot 10^{-7}$ & $5,3 \cdot 10^{-6}$ \\
\hline$W 2$ & $4,2567 \cdot 10^{-7}$ & $3,5 \cdot 10^{-6}$ \\
\hline
\end{tabular}

Wie aus Tab. 1 ersichtlich ist, wurde die höchste Protonenleitfähigkeit für den Kristalliten in Position $H$ (hochkant) ermittelt, obwohl der Kristallit in dieser Position die geringste Kontaktfläche auf der IDE aufweist. Dies lässt auf eine Vorzugsrichtung der Protonenleitfähigkeit schließen. Diese Anisotropie deutet darauf hin, dass die Poren der MOF-Struktur als Pfade für die Protonenleitung relevant sind.

Der Unterschied in den Protonenleitfähigkeiten ist geringer als erwartet [5]. Dies ist vermutlich darauf zurückzuführen, dass der untersuchte Kristallit Defekte bzw. Versatzstellen aufweist und somit weitere Effekte bei der Impedanzauswertung berücksichtig werden müssten. 


\section{Zusammenfassung}

In dieser Arbeit wurden erste Ergebnisse zur Entwicklung eines Sensorelements zur in situ Überwachung der Feuchte und Protonenleitfähigkeit in neuartigen Protonenaustauschmembranen (PEM) für Brennstoffzellen (FCs) auf Basis von impedanzspektroskopischen Untersuchungen vorgestellt. Das Hauptaugenmerk lag bei diesen Arbeiten auf einem geeigneten Aufbau zur Vermessung von Anisotropieeffekten sowie dem Einfluss von Temperatur und Feuchte.

Dazu wurde die Protonenleitung einzelner Kristallite neuartiger Materialien auf Basis von metallorganischen Gerüstverbindungen charakterisiert.

Es konnte gezeigt werden, dass die vorgestellten Materialien noch nicht die gewünschte Feuchteunabhängigkeit aufweisen. Der Protonenwiderstand der Kristallite zeigte eine deutliche Abhängigkeit von der eingestellten relativen Feuchte.

Zudem konnte auch gezeigt werden, dass eine Vorzugsrichtung für die Protonenleitfähigkeit existiert und somit Ansiotropieeffekte messbar sind.

\section{Literaturnachweis}

[1] S. M. Rezaei Niya, M. Hoorfar, Journal of Power Sources, 240, 281-293 (2013); doi: 10.1016/j.jpowsour.2013.04.011

[2] H. Furukawa, K. E. Cordova, M. O'Keeffe, O. M. Yaghi, Science, 341, 1230444-1-12 (2013); doi: 10.1126/science.1230444

[3] A.-L. Li, Q. Gao, J. Xu, X.-H. Bu, Coordination Chemistry Reviews, 344, 54-82 (2017); doi: 10.1016/j.ccr.2017.03.027

[4] O. Beyer, T. Homburg, M. Albat, N. Stock, U. Lüning, New J. Chem, 41, 8870-8876 (2017); doi: $10.1039 / C 7 N J 01697 B$

[5] D. Umeyama, S. Horike, M. Inukai, T. Itakura, S. Kitagawa, J. Am. Chem. Soc. 134 (2012) 12780-12785; doi: 10.1021/ja304693r

[6] T. Soboleva, X. Xie, Z. Shi, E. Tsang, T. Navessin, S. Holdcroft, J. Electroanal. Chem. 622, 145-152 (2008); doi:

10.1016/j.jelechem.2008.05.017 